\title{
Combining strengths to find high-quality leads
}

A comprehensive study prospectively comparing hits from high-throughput screening (HTS) and virtual screening of the same compounds against the protease cruzain - a target for Chagas disease - has shed light on the strengths and weaknesses of these two approaches and the potential for their integration.

HTS and virtual screening are often used independently to identify potential starting points for drug discovery, and each strategy has its own distinct flaws and virtues. However, direct comparisons between the two approaches are very rare. To provide such a comparison, Simeonov,

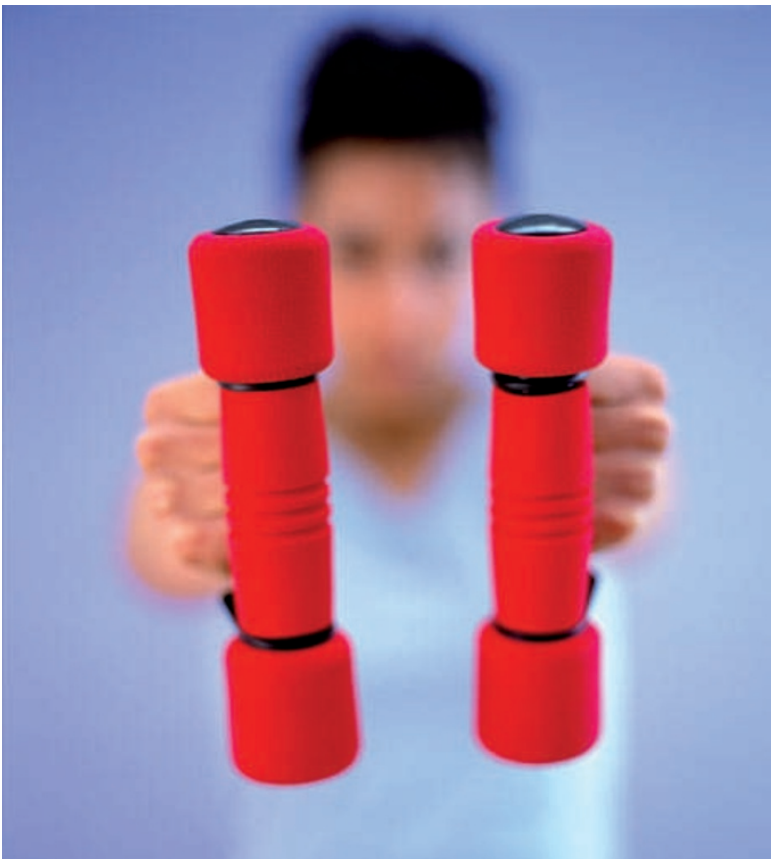

BANANA STOCK
Shoichet and colleagues conducted HTS of a 198,000-compound library to find inhibitors of cruzain, and in parallel performed a computational docking screen of the same compounds using the $\mathrm{X}$-ray structure of the protease.

After the initial removal of molecules that could rapidly be identified as artefacts from HTS, the authors investigated in depth the remaining 900 hits from the HTS, based on two parallel tracks. In one track, hits from HTS that also had high scores in the docking screen were pursued. In the second track, hits were followed up solely on the basis of chemotype clustering and behaviour in HTS.

Ultimately, various confirmatory experiments from these two tracks led to the identification of $146 \mathrm{com}$ pounds that were reversible, competitive inhibitors of cruzain (inhibition constants ranging from $65 \mathrm{~nm}$ to $6 \mu \mathrm{M}$ ), which represented five different classes. Two classes were discovered through the HTS track alone; that is, they were false negatives in the docking screen. Another two classes were only prioritized because of their high docking rank, and the final class was pursued on the basis of high docking scores and HTS evaluation.

The authors also conducted detailed mechanistic experiments, and determined the crystal structure of an inhibitor-cruzain complex (at $1.28 \AA$ resolution), to gain insights into the cause of false positives and false negatives in HTS and docking screening. It was shown that colloidal aggregation is the main source of false positives in HTS and this aggregation can be easily eliminated by the use of a prior screen using detergent. For docking screens, the authors highlight the importance of considering protein flexibility and other energetic forces when prioritizing compounds on the basis of the scores.

Overall, this study highlights the complementarity of HTS and virtual screening. In particular, integration of these two techniques can prioritize molecules that have desirable assay behaviour from those predicted to be active from docking screens and to show activity in HTS, thus limiting the impact of false positives from either strategy alone. Furthermore, it could aid the discovery of therapies for Chagas disease by providing novel reversible inhibitor chemotypes for cruzain, a target for which most known inhibitors are irreversible and covalent, and which are often viewed as being less tractable for development into drugs.

Man Tsuey Tse

ORIGINAL RESEARCH PAPER Ferreira, R. S et al. Complementarity between a docking and a high-throughput screen in discovering new cruzain inhibitors. J. Med. Chem. 53, 4891-4905 (2010)

FURTHER READING Schneider, G. Virtual screening: an endless staircase? Nature Rev. Drug Discov. 9, 273-276 (2010) 